\title{
Conrad Geßners Itinerar seiner Bündner Reise von 1561
}

\author{
Von Rudolf Steiger
}

Conrad Geßner (1516-1565) hat mehrere botanische Exkursionen in die Berge unternommen. Im gleichen Jahr 1541, in dem er sich in Basel den Doktorhut holte, hat er seinen Brief an Avienus veröffentlicht, worin er von seinem Vorhaben spricht, alljährlich einen oder mehrere Berge zu besteigen. Obschon die Liebe zu den Bergen und namentlich zu ihrer Pflanzenwelt ihn bis an das Lebensende begleitete, ist es ihm, dem ständig mit Arbeit Überhäuften und von Krankheit Behinderten, nicht möglich gewesen, sein Vorhaben in vollem Umfang auszuführen. Doch hat er den Pilatus bestiegen, die Glarner Berge wahrscheinlich mehrfach besucht, ebenso die Berge am Walensee und auf beiden Seiten des Seeztales, die Schwyzer Berge; dort hat er mehrmals besonders am Bockmattli botanisiert. Auf seiner Reise nach Venedig hat er den Splügen überquert und auf der Rückreise wahrscheinlich den Gotthard. Beschrieben hat er einzig seine Besteigung des Pilatus von 1555. Diese Beschreibung enthält mehrere erstmalige Beobachtungen, so über die Vegetationsstufen, und ist als frühester naturwissenschaftlicher Bericht über einen Schweizer Berg zu betrachten. Man bedauert, daß Geßner über seine anderen Alpenreisen keine zusammenhängenden Angaben hinterlassen hat. Ganz besonders vermissen wir eine genauere Beschreibung seiner größten Alpenreise, die ihn 1561 durch die Bündner Berge bis Bormio im oberen Veltlin geführt hat. Bisher waren von ihr nur spärliche Andeutungen in der gedruckten Korrespondenz bekannt, die nicht einmal genügen, daß man sich vom Reiseweg ein genaueres Bild machen kann.

Einige Autoren haben versucht, anhand der Fixpunkte, die sich aus den Briefen ergeben, den Reiseweg zu rekonstruieren. Der ausführlichste Versuch dieser Art findet sich in Christian Brüggers Ostrhätischen Studien zur Geschichte des Badelebens (Zürich 1863). Darin heißt es, es sei zwar keine Beschreibung der Reise aufbewahrt, aber ihre Richtung und Dauer lasse sich aus dem Briefwechsel bestimmen. Brügger nimmt an, Geßner sei zunächst ins Oberengadin gereist, um den mit ihm befreundeten Friedrich von Salis zu besuchen. Dabei sei er ohne Aufenthalt wahrscheinlich über den Julier, nicht über den Albula gezogen. In Samaden traf er Salis, der damals in Poschiavo weilte, nicht zu Hause an. Geßner habe sich in das Unterengadin begeben, in Schuls gebadet und dessen Quellen untersucht. Von da sei er über den Ofenpaß in das Münstertal und weiter über den Umbrailpaß nach den Bädern von Bormio gezogen, wo er einige Tage verweilte. Auf der Heimreise habe er den Weg durch das Veltlin genommen und sei 
wahrscheinlich über Chiavenna und den Splügenpaß nach Chur gelangt. Diese Darstellung Brüggers über Geßners Reiseweg wurde von Ernst Rolf Buser in seiner 1954 erschienenen Dissertation über die Entwicklung des Badewesens im Unterengadin wiederholt. Andere Verfasser, die auf die Reise Geßners zu sprechen kommen, bringen am mutmaßlichen Itinerar Geßners kleine Varianten in Vorschlag, so Traugott Schieß in seiner Biographie von Fabricius Montanus vom Jahre 1904 und Hans Fischer in seinem Aufsatz über Fabricius und Conrad Geßner (Zürich 1940). Beide nehmen an, Geßner habe auf dem Weg ins Engadin den Albula benutzt. Schieß stellt resigniert fest, daß uns Genaueres über den Weg, den Geßner und seine Begleiter auf der Hin- und Rückreise einschlugen, nicht bekannt sei. Die älteren Biographien Geßners (Simler, Schmiedel und Hanhart), denen bestenfalls auch nur die gleichen Quellen wie den neueren zugrunde gelegt sind, bringen naturgemäß nicht mehr Licht in das Geheimnis.

Bei dieser Sachlage waren weitere Einsichten nur von Quellen zu erwarten, die in bezug auf die vorliegende Frage bisher keine Auswertung erfahren haben. Mein Freund Dr. Charles Salzmann hat mich auf die Bullingerkorrespondenz mit den Graubündnern aufmerksam gemacht. Dieser wertvolle Hinweis hat den Anstoß zu der vorliegenden Untersuchung gegeben. Die Quellen, die ich verwendet habe, sind:

1. Bullingers Korrespondenz mit den Graubündnern, herausgegeben von Traugott Schieß, 3 Bände, Basel 1904-1906.

2. Geßners «Liber amicorum», das sich in der National Library of Medicine, Bethesda, Maryland, USA, befindet und wovon die Zentralbibliothek Zürich eine Photokopie besitzt.

3. Die Legenden auf Geßners Pflanzenbildern, deren Originale Eigentum der Universitätsbibliothek Erlangen sind. Auch von diesen konnte ich eine Photokopie in der Zentralbibliothek Zürich benützen.

4. Geßners Nachträge zu seinen «Horti Germaniae» von 1561, die in diesem Werk beigedruckt sind.

5. Johannes Bauhins posthum veröffentlichte «Historia plantarum universalis», Yverdon 1651. (Diese für unseren Gegenstand wichtige Quelle wurde bisher nie in dieser Absicht befragt.)

Außerdem habe ich mich folgender Hilfsmittel bedient:

- Aegidius Tschudi, De prisca et vera Alpina Rhaetia, Basel 1538.

- Johannes Petrus Guler von Weineck, Rhaetia, Zürich 1616.

Ferner wurden einige moderne Reisehandbücher von Graubünden, Abhandlungen über den bündnerischen Bergbau und die bündnerische Flora beigezogen. 
Über die Vorgeschichte der Reise konnten folgende Angaben beigebracht werden. Im November 1557 ersuchte Bullinger den Fabricius Montanus, von Friedrich von Salis eine Beschreibung des Wormserbades zu erbitten. Im Dezember dieses Jahres erklärt Geßner in einem Brief an Fabricius, er gedenke, sobald er das Fischbuch abgeschlossen habe, das Wormserbad (Bormio) zu besuchen. Im Juni 1560 schreibt Geßner an Johannes Bauhin, er habe die Bündner Reise noch nicht antreten können wegen Erkrankung seiner Frau. Er habe im Sinn, eine Abhandlung über die schweizerischen und deutschen Bäder zu schreiben. Aus einem Brief Geßners an Fabricius vom 13. April 1561 geht hervor, daß bereits feststand, Fabricius werde Geßner in die Bäder von Bormio begleiten. Die Abreise war damals auf Anfang August vorgesehen. Sie wurde später vorverlegt. Am 13. Juni schreibt Bullinger einen Brief, den Geßner Fabricius überbringen sollte.

Geßner ist am 14. Juni abgereist, denn wie aus einem Schreiben des Fabricius an Bullinger hervorgeht, kam er am 15. Juni abends in Chur an. (Für die Reise Zürich-Chur brauchte man damals zwei Tage.) Im gleichen Brief vom 16. Juni berichtet Fabricius, er gedenke am folgenden Tag in Begleitung Geßners und des Bürgermeisters Johann Tscharner von Chur die Reise nach Bormio über das Veltlin anzutreten. Diese wichtige Angabe ist bisher nicht beachtet worden, geht doch aus ihr mit großer Wahrscheinlichkeit hervor, daß das Veltlin zur Hinreise und nicht auf dem Rückweg benutzt werden sollte. Daß dieser Plan wirklich ausgeführt wurde, ist in einem Schreiben Geßners an Crato vom 16. August 1561 bezeugt. Dort steht, seine Alpenreise habe ihn bis in den obersten Teil des Veltlins und zu den Wormser Thermen geführt.

Bei einer seiner Pflanzenzeichnungen bemerkt Geßner: «invenit Jo. Bauhinus cum mecum esset ad pedem Septimi montis (ubi pernoctavimus) »: Johannes Bauhin hat sie gefunden, als er mit mir am Fuße des Septimer war, wo wir übernachtet haben. In den Nachträgen zu den «Horti Germaniae» wird von einer Pflanze gesagt, Geßner habe sie auch gesehen «infra Septimum montem initio Pregaliae vallis »: unterhalb des Septimer, wo das Bergell anfängt. In seiner «Historia plantarum universalis» erklärt Bauhin, er habe eine gewisse Pflanze auf dem Septimer im Juni blühend angetroffen. Aus diesen Zitaten, die leicht vermehrt werden könnten, geht hervor, daß Geßner und seine Begleiter im Juni den Septimerpaß von Norden her überschritten haben, nachdem sie wahrscheinlich in Bivio übernachtet hatten.

In Chiavenna hat Geßners Reisegesellschaft übernachtet und wahrscheinlich einen Ruhetag eingeschaltet, denn Geßner hat hier Zeit gefunden, den Garten des Franciscus Portus, eines Philologen, der glaubenshalber in Chiavenna Zu- 
flucht gefunden hatte, zu besichtigen. Auch muß er einen Abstecher nach Plurs gemacht haben, denn er schreibt in seinem Handexemplar von Mattiolis Dioskorideskommentar, daß er dort eine gewisse Pflanze gesehen habe. Wie aus der Bullingerkorrespondenz hervorgeht, hatte Fabricius als Dekan der Bündner Synode in Chiavenna verschiedene Geschäfte zu erledigen.

In der Bullingerkorrespondenz und in einem Brief Bauhins an Geßner finden sich Stellen, die beweisen, daß Geßner, Fabricius und Bauhin in Teglio von dem reformierten Arzt Nikolaus Guicciardi gastfreundlich aufgenommen wurden. Dieser Arzt hat sich wohl bei dieser Gelegenheit in Geßners "Liber amicorum» eingetragen. Bürgermeister Tscharner, der ja auch zur Reisegesellschaft gehörte, ist möglicherweise nach Tirano, dem Sitz des bündnerischen Probstes für das Veltlin, weitergeritten, wo er vielleicht in der Probstei beherbergt wurde.

In Tirano müssen die Reisenden einen Marschhalt eingeschoben haben, denn Fabricius hatte auch hier kirchliche Geschäfte zu erledigen und mit der Probstei zu sprechen. Geßner bemerkt zu der Abbildung einer seltenen Pflanze, er habe sie nirgends als beim Apotheker zu Tirano gesehen. Vielleicht ist dieser Apotheker identisch mit einem gewissen Ludwig Quadrio, einem überzeugten Anhänger der Reformation, dessen Sohn damals die Zürcher Schule besuchte.

Das Endziel der Reise, die Bäder von Bormio, hat Geßner wahrscheinlich um den 23. Juni herum erreicht. In seinem Brief an den englischen Arzt John Caius erklärt er, daß Bormio acht Tagereisen von Zürich entfernt sei. Die teils gesicherten, teils mutmaßlichen Rastorte der Hinreise sind somit:

Weesen. Geßner bemerkt in seinen Emendanda zu Valerius Cordus, dieser Ort sei eine Tagereise von Zürich entfernt. In der nächsten Umgebung von Weesen haben Geßner und Bauhin botanisiert, wie sich aus einigen Pflanzenfunden ergibt. Zu einem dieser Funde bemerkt Bauhin ausdrücklich, er sei im Juni gemacht worden. Dies will sagen: auf der Hinreise und nicht etwa auf der Rückreise, die wahrscheinlich ebenfalls durch Weesen führte, aber erst im Juli.

Der Rastort des zweiten und dritten Tages war offenbar Chur. Am Ruhetag, das heißt am 16. Juli, wurde, wie aus einigen datierten Pflanzenfunden zu schlieBen ist, zwischen Chur und dem Rhein botanisiert und auch dem Gärtlein von Fabricius ein Besuch abgestattet, worin dieser Alpenpflanzen zog. Am gleichen Tag haben sich von Geßners Churer Freunden im «Liber amicorum » eingetragen : Fabricius, der Pfarrer Philippus Gallitius und der Arzt Hieronymus Brixius; dieser mit dem Spruch : «Nihil tam difficile quaerendo investigari possit» (Nichts ist so schwierig, daß es nicht erforscht werden könnte).

Der nächste bezeugte Rastort muß sich, wie wir früher erwähnten, am Fuße des Septimerpasses, wahrscheinlich in Bivio, befunden haben. Dieser Ort ist aber 
so weit von Chur entfernt, daß er wahrscheinlich in zwei Etappen erreicht wurde. Vielleicht haben die Reisenden die Nacht vom 17. auf den 18. Juni in Tinzen verbracht, wo sich nach zeitgenössischen Quellen eine Sust befand. Die Strecke von Tinzen nach Bivio (15 km, $544 \mathrm{~m}$ Steigung) konnte am Vormittag zurückgelegt werden, so daß Geßner und Bauhin der Nachmittag zum Botanisieren am Fuße des Septimer, also in der Umgebung von Bivio, zur Verfügung stand.

Die Strecke von Bivio nach Chiavenna konnte in dem damals üblichen Tagesritt von 10 Stunden zurückgelegt werden. Unsere Reisenden könnten also am Abend des 20. Juni in Chiavenna angekommen sein. Hier müssen sie, wie oben gezeigt wurde, einen Ruhetag eingeschaltet haben. Am 22. Juni, einem Sonntag, dürften sie die Reise fortgesetzt haben.

Der nächste einigermaßen bezeugte Rastort war Teglio. Weil die Strecke von Chiavenna bis Teglio (75 km) für einen normalen Tagesritt zu groß war, dürfte auch dieser Rastort in zwei Etappen erreicht worden sein. Die Nacht vom 22. auf den 23. Juni könnten unsere Reisenden in Morbegno verbracht haben.

Wahrscheinlich haben Geßner und seine Begleiter Teglio am 24. Juni verlassen und nach einem kurzen Zwischenhalt in Tirano noch am selben Tag Bormio erreicht. Wenn wir die gesicherten und die mutmaßlichen Rastorte Geßners zusammenzählen, kommen wir auf die acht Tage, in denen nach Geßners Aussage Bormio von Zürich aus zu erreichen ist.

Der Aufenthalt in Bormio dürfte genau eine Woche gedauert haben. In ihr wurden die Bäder benutzt, und vor allem wurde in deren Umgebung eifrig botanisiert. Ich habe unter den Pflanzenbildern Geßners 15 gefunden, bei denen als Fundort Bormio vermerkt war. Bauhin verzeichnet in seiner «Historia plantarum universalis» etwa die gleiche Anzahl von Pflanzen, darunter andere als die von Geßner genannten, die er von Bormio heimgebracht habe. Bei einem Pflanzenbild vermerkt Geßner: «Vide an hanc aut simillimam viderim Burmii in prato montano: cum Joannes Bauhinus mecum esset et loci illius chirurgus» (Siehe nach, ob ich diese oder eine sehr ähnliche Pflanze zu Bormio auf einer Bergwiese gefunden habe, als Johannes Bauhin und der Chirurg des Ortes mich begleiteten). Ohne Zweifel verweist er hier auf sein Herbar, in dem die zu vergleichende Pflanze aufbewahrt wurde. Es ist dies wohl als Beweis zu werten, daß Geßner (und wahrscheinlich auch Bauhin) die auf der Reise gesammelten Pflanzen preßten und später ihrem Herbar einverleibten. Als Monat, in dem diese in Bormio gesammelten Pflanzen gefunden wurden, ist immer der Juni angegeben, ein Fingerzeig, daß sich der Aufenthalt an diesem Badeort nicht bis in den Juli hinein erstreckt hat. In einem ganz vereinzelten Fall ist der Juli als Fundmonat angegeben; doch dürfte dies auf einem Versehen beruhen. 
Als Fundort vieler gesammelter Pflanzen ist sowohl bei Geßner wie bei Bauhin der Mons Braulius genannt. Darunter ist nach damaligem Sprachgebrauch der Umbrailpaß zwischen Bormio und Santa Maria im Münstertal zu verstehen und nicht der erst im 19. Jahrhundert als Monte Braulio bezeichnete Berg an diesem Paß. Der Aufstieg zum Umbrail wird in der «Guida della Valtellina » von 1873 als «ricco di fiori e piante rarissime e preziose pel botanico» bezeichnet. Ich vermute, daß unsere Reisegesellschaft den $\mathrm{Pa}$ zu Fuß überschritten hat, um diese botanischen Schätze besser einheimsen zu können. Außer Geßner und Bauhin war nämlich auch Fabricius ein großer Pflanzenliebhaber. Als Fundmonat der hier gefundenen Pflanzen wird bald der Juni, bald der Juli angegeben. Einmal sagt Geßner von einer Pflanze, er habe sie «copiosam in summo Monte Braulio sub finem Junii florentem» (zahlreich auf der Paßhöhe des Umbrail Ende Juni blühend) angetroffen. Es liegt deshalb nahe, anzunehmen, die Überschreitung des Umbrail habe am 30. Juni und 1.Juli stattgefunden, wahrscheinlich mit Übernachtung in dem nach Guler von Weineck schon damals auf der Paßhöhe befindlichen Gasthaus.

Die Ermittlung des nachfolgenden Rastortes bereitet Schwierigkeiten. Als einziger Anhaltspunkt liegt Geßners Fundortsangabe einer von ihm abgebildeten Pflanze vor. Sie lautet: «Reperi imm Engadin bimm bergwerck ubi quidam Germanicè loquentes Hundsbeere vocabant» (Ich habe sie im Engadin beim Bergwerk gefunden, wo einige deutsch Sprechende sie Hundsbeere nannten). Als einzige Bergwerke, die damals im Engadin in Betrieb standen, kommen jenes am Ofenpaß und jenes bei S-charl im S-charl-Tal in Betracht. Bei beiden befanden sich wohl auch Unterkunftsstätten. Die Strecke von der Paßhöhe des Umbrail bis zum Gasthaus des Ofenpasses kann in etwa acht Marschstunden, diejenige vom Umbrail bis S-charl in etwa elf Stunden zurückgelegt werden. Der Weg über S-charl ist der direktere nach Scuol (13 Wegstunden), derjenige über den Ofenpaß (14 Wegstunden) ein kleiner Umweg. In bezug auf Gangbarkeit war der Weg über den Ofenpaß wohl ratsamer. Ich möchte deshalb annehmen, daß Geßner diesen gewählt hat. Leider läßt Geßners beim Bergwerk gefundene Pflanze keine Schlüsse zu. Nach seiner Abbildung handelt es sich um die Lonicera nigra, die sowohl um den Ofenpaß wie bei S-charl - hier allerdings anscheinend häufiger vorkommt. Daß Geßner beim Bergwerk deutsch sprechende Leute (wohl tirolische Bergknappen) angetroffen hat, konnte bei beiden Bergwerken der Fall sein. Bauhin bemerkt bei zwei Pflanzen, er habe sie «in monte prope Münsterthal» gefunden. Dieser Ausdruck kann ebensogut den Ofenpaß wie den CostainasPaß bedeuten.

Als Endpunkt der nächsten Etappe ist Scuol (Schuls) anzusehen, wo unsere 
Reisenden wohl am Abend des 2. Juli angekommen sind. Auch hier wurde botanisiert; aber in der Hauptsache galt der Besuch einer Trinkkur bei den dortigen Heilquellen. Geßner hat das Wasser dieser Quellen, das er als sehr heilkräftig einschätzte, ausgekocht und die Rückstände untersucht. Der Aufenthalt in Schuls dürfte nur zwei Tage gedauert haben.

Der nächste Rastort dürfte Süs gewesen sein, denn auch von hier werden Pflanzenfunde registriert. Vielleicht genossen unsere Reisenden die Gastfreundschaft des Ortspfarrers Ulrich Campell, der Geßner noch im gleichen Jahr Pflanzen schickte. Campell ist der Verfasser einer wertvollen Beschreibung Graubündens (Raetiae Alpestris topographica descriptio), die erst 1884-1890 gedruckt worden ist. Darin ist der Besuch Geßners und des Fabricius in Schuls in einer Weise erwähnt, daß eine persönliche Erinnerung daran unverkennbar ist.

Von Süs ging die Weiterreise bis zum nächsten Rastort. Als solcher ist Zuoz oder Ponte oder noch wahrscheinlicher Samaden anzunehmen, wo man wahrscheinlich den mit Geßner und Fabricius befreundeten Humanisten und Staatsmann Friedrich von Salis anzutreffen hoffte. Dieser war aber gerade in jenen Tagen im Puschlav abwesend. In Samaden lebte auch der mit Geßner seit Jahren korrespondierende Jurist Jakob Bifrun, der als erster eine Grammatik der romanischen Sprache verfaßt hatte und von Geßner in seinem «Mithridates» von 1555 deshalb gelobt worden war.

Von hier führte die Reise über den Albulapa $\beta$. Diese Route ist durch zwei Pflanzenfunde Bauhins gesichert. Der einen dieser Pflanzen gedenkt Bauhin mit den Worten: «Hanc in peregrinatione mea Rhaetica cum Domino Gesnero in monte Albula reperi, florentem Junio» (Diese Pflanze habe ich auf meiner Reise durch Graubünden mit Herrn Geßner auf dem Albula im Juni blühend gefunden). Das Wort Juni muß aus «Juli» verschrieben sein, wie aus der ganzen Situation hervorgeht. Vom zweiten Pflanzenfund auf dem Albula ist ausdrücklich gesagt, daß er im Juli gemacht wurde.

Als nächster Rastort wäre Bergün zu erwarten gewesen, wo die meisten Wanderer über den Albula die Nacht zu verbringen pflegten. Aber Geßners Interesse für Heilbäder könnte den Ausschlag gegeben haben, daß unsere Reisenden wahrscheinlich bis Alvaneu weiterritten, wo sich ebenfalls Unterkunftshäuser befanden. Für Alvaneu als Rastort scheinen nämlich von Geßner erwähnte Pflanzenfundorte zu sprechen. Eine Erwähnung lautet: «Nuper Bauhinus noster, peregrinationis per alpes Rhaeticas mihi comes, in itinere non procul Alvinovo fonte sulfureo sibi inventam ostendit virentem» (Bauhin, mein Begleiter auf der Exkursion nach Graubünden, hat mir ein von ihm auf der Reise nicht weit von der Schwefelquelle Alvaneu gefundenes frisches Exemplar der Pflanze gezeigt). 
Eine Stelle in der Bullingerkorrespondenz scheint darauf hinzudeuten, daß mindestens Fabricius schon am folgenden Tag wieder in Chur weilte, wo ihn vielleicht dringende Geschäfte erwarteten. Es ist nicht unwahrscheinlich, daß Geßner und Bauhin noch einen Tag in Alvaneu geblieben sind: Geßner, um die dortigen Heilquellen zu untersuchen, Bauhin, um zu botanisieren.

Von Alvaneu an ist der weitere Verlauf der Rückreise mangels sicherer Anhaltspunkte schwierig festzustellen. Möglicherweise sind die beiden Botaniker das Landwasser hinauf nach Davos geritten. Hier amtete als Pfarrer seit kurzem Thomas Egli, der als Zögling der Zürcher Schule Geßner wohlbekannt war. Es ist auffällig, daß Egli nach der Bündner Reise Geßners plötzlich als dessen Pflanzenlieferant für die Flora von Davos erscheint. Bei mehreren Pflanzenbildern Geßners, wo Davos als Fundort vermerkt ist, bleibt es ungewiß, ob er die Pflanzen selbst gesammelt oder von Egli erhalten hat.

Von Davos wird die Reise über Klosters ins Prättigau gegangen sein, wo Geßner als Gast eines Pfarrers Johannes Marius die nächste Nacht verbrachte. Welche Kirche jener versah, ist nicht überliefert; aber sicher ist, daß dieser Pfarrer ihn später mit Pflanzen aus dem Prättigau beliefert hat. Bauhin registriert einen Pflanzenfund nicht weit vom Bad Fideris, den er allerdings in den Juni datiert. Aber es ist keineswegs sicher, daß dieser Fund sich auf diese Reise bezieht, denn Bauhin ist später noch durch jene Gegend gekommen. Da aber Geßner seine Reise auch zu dem Zweck unternommen hatte, Stoff für sein Bäderbuch zu sammeln, ist es nicht ganz ausgeschlossen, daß er auch dieses Bad aufgesucht hat, obschon er es von früher her kannte. Mithin könnte sich in Malans oder Marschlins ein Rasttag eingeschaltet haben.

Der letzte Rastort vor Zürich dürfte Weesen gewesen sein. Daß hier wieder botanisiert wurde, geht aus einigen Pflanzenfunden Bauhins hervor. Bei einem derselben steht der Vermerk: «Hanc cum Gesnero in peregrinatione nostra observavimus in monte prope Wesen ... florentem mense Julio» (Diese Pflanze sah ich bei der Reise mit Geßner auf einem Berg bei Weesen im Juli blühend).

Um den 13. Juli herum dürften die Reisenden in Zürich angekommen sein, denn Geßner sagt mehrmals, die Reise habe ungefähr einen Monat gedauert.

Auf der Reise hatte Geßner für sein geplantes großes Pflanzenwerk weitere bis dahin unbekannte Alpenpflanzen aufzufinden gehofft. Auch wollte er eine stark vermehrte Neuauflage seiner 1553 erschienenen Abhandlung über die Bäder Helvetiens und Germaniens vorbereiten. Über den Zweck seiner Reise äußert sich Geßner am ausführlichsten in einem nach der Rückkehr geschriebenen Brief an John Caius. Darin heißt es: «Neulich war ich so ziemlich einen Monat lang abwesend, indem ich bis zu jenen zahlreichen und sehr hohen Bündner Bergen 
vordrang, die Helvetien von Italien trennen. Sie sind etwa acht Tagereisen von Zürich entfernt. Ich wollte gesundheitshalber einige dort befindliche berühmte Heilquellen benutzen und bei dieser Gelegenheit auch ihre Beschaffenheit erforschen. Außerdem hatte ich mir vorgenommen, zu botanisieren, alte Freunde zu besuchen und neue zu gewinnen, meinen Geist von den Studien sich erholen zu lassen und meinen Körper zu trainieren. All dies ist, Gott sei Dank, ordentlich gelungen.»

\section{Anhang}

\section{Liste der Zitate}

- Geßners Notiz über einen Pflanzenfund am Septimer

- Eintrag des Brixius in Geßners "Liber amicorum»

- Geßners Eintrag bei der Pflanze von der Bergwiese bei Bormio

- Zitat «ricco di fiori»

- Geßners Notiz vom Pflanzenfund auf der Umbrail-Paßhöhe

- Geßners Notiz von der Hundsbeere bimm bergwerck

- Bauhins Notiz über einen Pflanzenfund auf dem Albula

- Geßners Erwähnung von Bauhins Pflanzenfund bei Alvaneu

- Bauhins Notiz über einen Pflanzenfund bei Weesen

- Brief Geßners an Caius (nur Übersetzung)

\section{Route}

Zürich-Weesen-Chur-Tinzen-Bivio-Septimerpaß-Chiavenna-Morbegno-Teglio-TiranoBormio-Umbrailpaß-Ofenpaß-Schuls-Süs-Samaden-Albulapaß-Alvaneu-Davos-Prättigau-Weesen-Zürich

\section{Anmerkung}

Dr. Rudolf Steiger (1896-1978) hat diesen Vortrag am Tag vor seinem 73. Geburtstag im Mai 1969 an der Sigerist-Tagung in Morges gehalten. Auf einer beim Verlag Orell Füßli geliehenen Bündner Schulwandkarte hatte er das Itinerar markiert. Die aus Geßners Korrespondenz bekannten Fixpunkte waren mit blauen Aufklebescheibchen vermerkt (Chur, Teglio, Bormio, Schuls, Samaden). Die aus dem weiteren Stoff erschlossenen Etappen hatte er mit roten Scheibchen hervorgehoben (Weesen, Bivio, Chiavenna, Ofenpaß, Süs, Alvaneu, Davos, Prättigau). Die auf Grund der Wegstrecken berechneten, aber durch keine Belege gesicherten Rastorte trugen weiße Scheibchen (Tinzen, Morbegno); halb weiß, halb rot waren Süs, Davos und Prättigau bezeichnet. Klebepfeile gaben den Richtungssinn an. Auf meine spätere Bitte, er solle den Vortrag im Gesnerus veröffentlichen, antwortete Rudolf Steiger, es seien noch nicht alle Punkte abgeklärt. In seinem Nachlaß auf der Zentralbibliothek Zürich suchten Georg Bührer und Frl. Margaret Eschler nach dem gewünschten Vortragsmanuskript und brachten es wieder zum Vorschein. Es war druckreif getippt und trug hie und da später von Hand nachgetragene Abänderungen, die natürlich hier berücksichtigt sind.

Heinz Balmer 


\section{Summary}

Different authors believed that Conrad Gessner, in his great Alpine journey of 1561, travelled from Zürich to the Engadine, that he went from there by the Ofen- and UmbrailPass to Bormio and returned through the Valtellina. Steiger proves that Gessner travelled in the opposite direction. He tries to make out his progression day by day. His main sources are Bullinger's correspondence and some plant descriptions by Gessner and by his fellowtraveller Johannes Bauhin.

Dr. phil. Rudolf Steiger $\dagger$

Rüterwiesstraße 18

CH-8125 Zollikerberg bei Zürich 